\title{
Evaluation of local strength via microstructural quantification in a pearlitic rail steel deformed by simultaneous compression and torsion
}

Nikas, Dimitrios; Zhang, Xiaodan; Ahlström, Johan

Published in:

Materials Science and Engineering: A - Structural Materials: Properties, Microstructure and Processing

Link to article, DOI:

10.1016/j.msea.2018.09.067

Publication date:

2018

Document Version

Peer reviewed version

Link back to DTU Orbit

Citation (APA):

Nikas, D., Zhang, X., \& Ahlström, J. (2018). Evaluation of local strength via microstructural quantification in a pearlitic rail steel deformed by simultaneous compression and torsion. Materials Science and Engineering: $A$ Structural Materials: Properties, Microstructure and Processing, 737, 341-347.

https://doi.org/10.1016/j.msea.2018.09.067

\section{General rights}

Copyright and moral rights for the publications made accessible in the public portal are retained by the authors and/or other copyright owners and it is a condition of accessing publications that users recognise and abide by the legal requirements associated with these rights.

- Users may download and print one copy of any publication from the public portal for the purpose of private study or research.

- You may not further distribute the material or use it for any profit-making activity or commercial gain

- You may freely distribute the URL identifying the publication in the public portal 


\section{Author's Accepted Manuscript}

Evaluation of local strength via microstructural quantification in a pearlitic rail steel deformed by simultaneous compression and torsion

Dimitrios Nikas, Xiaodan Zhang, Johan Ahlström

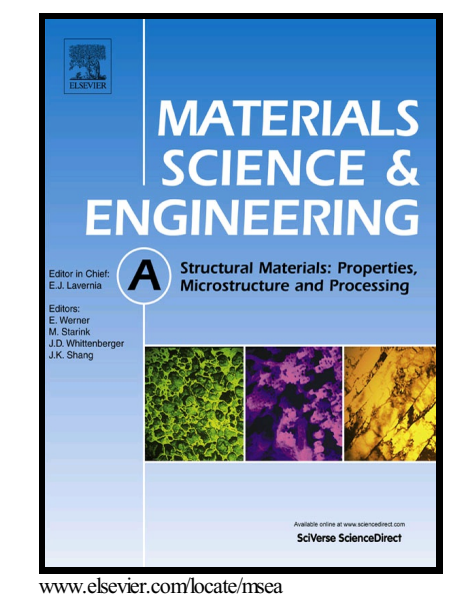

PII: $\quad$ S0921-5093(18)31273-5

DOI: $\quad$ https://doi.org/10.1016/j.msea.2018.09.067

Reference: MSA36948

To appear in: Materials Science \& Engineering A

Received date: 9 July 2018

Revised date: 24 August 2018

Accepted date: 18 September 2018

Cite this article as: Dimitrios Nikas, Xiaodan Zhang and Johan Ahlström, Evaluation of local strength via microstructural quantification in a pearlitic rail steel deformed by simultaneous compression and torsion, Materials Science \& Engineering A, https://doi.org/10.1016/j.msea.2018.09.067

This is a PDF file of an unedited manuscript that has been accepted for publication. As a service to our customers we are providing this early version of the manuscript. The manuscript will undergo copyediting, typesetting, and review of the resulting galley proof before it is published in its final citable form. Please note that during the production process errors may be discovered which could affect the content, and all legal disclaimers that apply to the journal pertain. 


\title{
Evaluation of local strength via microstructural quantification in a pearlitic rail steel deformed by simultaneous compression and torsion
}

\author{
Dimitrios Nikas ${ }^{a * \dagger}$, Xiaodan Zhang ${ }^{\mathrm{b}, \dagger}$, Johan Ahlström ${ }^{\mathrm{a}}$ \\ ${ }^{a}$ Division of Engineering Materials, Department of Industrial and Materials Science, Chalmers University of Technology, \\ 41296 Gothenburg, Sweden \\ ${ }^{b}$ Section of Manufacturing Engineering, Department of Mechanical Engineering, Technical University of Denmark \\ 2800 Kgs. Lyngby, Denmark
}

\begin{abstract}
Pearlitic steels are commonly used for railway rails because they combine good strength and wear properties. During service, the passage of trains results in large accumulation of shear strains in the surface layer of the rail, sometimes leading to crack initiation. Knowledge of the material properties versus the shear strain in this layer is therefore important for fatigue life predictions. In this study, fully pearlitic R260 rail steel was deformed using a bi-axial torsion-compression machine to reach different shear strains. Microstructural parameters including interlamellar spacing, thickness of ferrite and cementite lamellae and dislocation density in the ferrite lamellae, as well as hardness were quantitatively characterized at different shear strain levels. Based on the microstructural observations and the quantification of the microstructural parameters, the local flow stresses were estimated based on boundary strengthening and dislocation strengthening models. A good agreement was found between the estimated flow stresses and the flow stresses determined from microhardness measurements.
\end{abstract}

Keywords: Pearlitic rail steel, TEM, hardness, strengthening mechanisms, dislocation density

\section{Introduction}

The most common material type for manufacturing of rails nowadays are carbon steels with a predominantly pearlitic microstructure. Pearlite is a lamellar microstructure consisting of colonies of similarly oriented cementite lamellae embedded in a softer ferrite matrix. Steels with this microstructure exhibit a very good combination of wear and strength properties suitable for railway applications. In this paper the grade R260 is studied, which is one of the most common rail steel grades in Europe.

The surface layers of rails are subjected to very high rolling contact loads during their service life, which lead to large plastic deformation due to accumulation of large shear strains close to the running surface [1, 2]. These surface layers have typically position-dependent thickness from several micrometers to a few tens of millimeters and thus varying strain gradients, which limits the possibilities for a systematic study of the mechanical response of the layers. To understand the material behavior and enable large scale testing of the material, specimens with a fairly uniform microstructure within the test volume are required. If the microstructure observed in field including the gradient microstructure, could be recreated artificially in the lab, it would be possible to perform a thorough investigation of how the microstructure evolves with loads and of how the microstructure affects the mechanical properties.

To this end a method was developed to pre-deform test bars using a bi-axial test frame by combining compression and torsion, so that a sufficient strain level in the bars was achieved [3]. The comparison between the field samples and the test bars in the above study was performed by macroscopic $2 \mathrm{D}$ strain calculations such as the flow line method (see more details in [3]). However, the full picture such as the relationships among shear strain, microstructure, and strength, which can provide the baseline for the understanding of mechanical properties in the lab/field, is still unclear.

For the understanding of the mechanical properties such as flow stress/strength in deformed pearlitic steel [4-6] and other metals and alloys [7-9], microstructural characterization and quantification by scanning electron microscopy (SEM) and transmission electron microscopy (TEM) followed by computations of mechanical properties have been reported as powerful tools. A similar strategy will therefore be applied in the present study.

The main goal of the investigation is to characterize the artificially produced microstructure of predeformed test bars using SEM and TEM, to quantify the microstructural parameters such as interlamellar spacing (ILS), thickness of ferrite and cementite lamellae, and the dislocation density in the ferrite lamellae, and to correlate these parameters to the shear strains and the mechanical properties. The shear strains will be theoretically calculated at the different positions in the test bars from where the samples were extracted. 


\section{ACCEPTED MANUSCRIPT}

Strengthening mechanisms will be proposed based on the microstructural characterization and the structurestrength relationship will be suggested. The flow stresses will be calculated based on the quantification of microstructural parameters and compared with the measured ones determined from microhardness. The analysis can provide the knowledge for reliable evaluation of the local strength in field samples.

\section{Material and experimental}

\subsection{Sample extraction}

The material studied in this investigation is the railway rail steel grade R260. The microstructure of this material is almost fully pearlitic with nominal chemical composition as shown in table 1.

\begin{tabular}{|c|c|c|c|c|c|c|c|c|c|}
\hline $\mathrm{C}$ & $\mathrm{Si}$ & $\mathrm{Mn}$ & $\mathrm{P}$ & $\mathrm{S}$ & $\mathrm{Cr}$ & $\mathrm{Al}$ & $\mathrm{V}$ & $\mathrm{N}$ & $\mathrm{Cu}$ \\
\hline 0.72 & 0.31 & 1.04 & 0.006 & 0.010 & 0.02 & $<0.002$ & $<0.005$ & 0.006 & 0.018 \\
\hline
\end{tabular}

Table 1: \% mass composition of the R260 steel, analyzed according to ASTM E 572-13

Samples for characterization were extracted from test bars that were pre-strained in a bi-axial machine using torsion-compression. The test bars used for pre-deformation with the dimension as shown in Fig. 1, were turned from material extracted about $20 \mathrm{~mm}$ below the surface of virgin rail heads. A smooth transition radius of $100 \mathrm{~mm}$ was used to minimize the strain concentration at the end of the gauge section. The torsional stroke of the load frame is $90^{\circ}$, and hence a cyclic pre-deformation sequence was used. The predeformation experiments procedure and the initial comparison and characterization have been further described in a previous study [3]. The bars used for this investigation were twisted 3 times $\left(3 \times 90^{\circ}\right)$ and 7 times $\left(7 \times 90^{\circ}\right)$ using $500 \mathrm{MPa}$ nominal compression load (i.e. constant force, not increasing as the diameter increases), separately. The samples used for the microscopy and hardness measurement were taken from the very middle part of the gauge section of the bar. They were mechanically ground and polished to $0.04 \mu \mathrm{m}$ using colloidal silica suspension. Etching was done using $\mathrm{Nital}\left(3 \% \mathrm{HNO}_{3}\right.$ in ethanol).

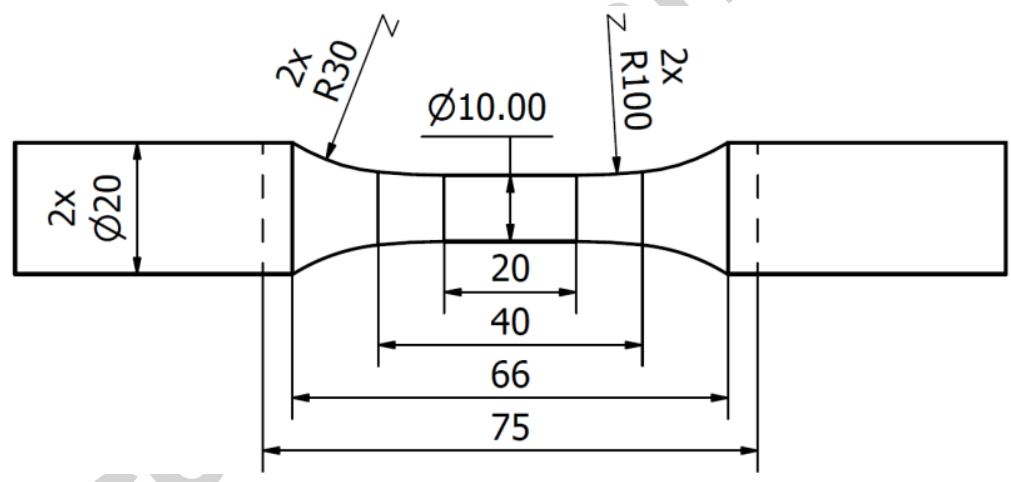

Figure 1: Test bar geometry (in $\mathrm{mm}$ )

\subsection{Strain calculation}

Simple shear is a good model to calculate shear strain in pure torsion tests [10]. It has been used also by others for example in high pressure torsion experiments [11]. In such experiments, the length of the twisted volume remains constant. In the tests now performed, the length of the test bar decreases due to the axial compressive force. Thus, a surface pattern consisting of a regular grid was imprinted on the specimens before the test, and the angle between these lines was then used to compute the shear strain of the test bars at different positions according to the following formula:

$$
\gamma=\frac{\tan (90-\theta)}{R} r
$$

where $\gamma$ is the angle measured at the outer surface of the test bars (see Fig. 2), $r$ is the radius measured from the center line of the bars and $R$ is the final radius of the test bars after the end of the pre-deformation procedure. 


\section{ACCEPTED MANUSCRIPT}

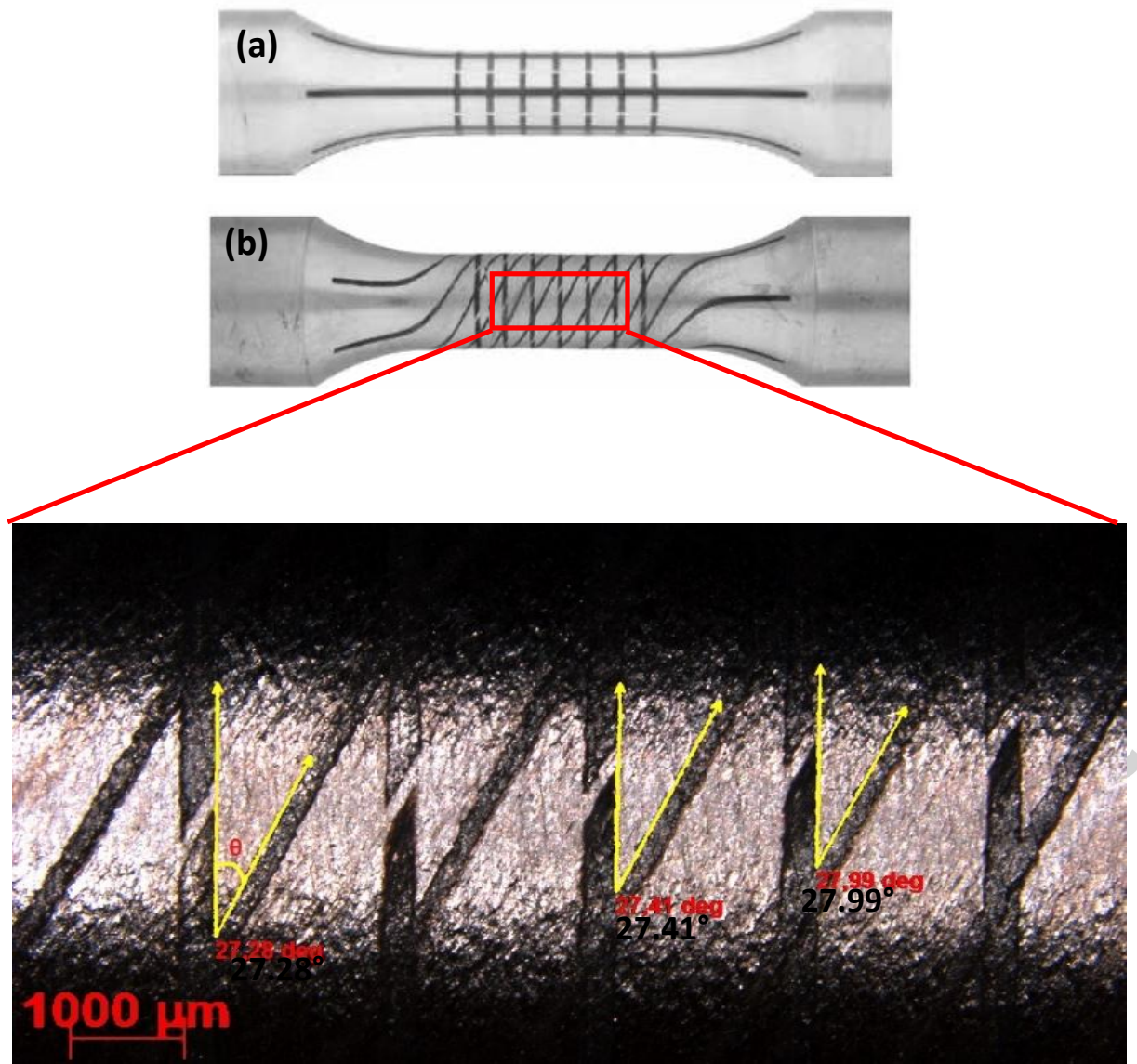

Figure 2: Calculation of shear strain from outer surface of a test bar. Parallel lines are engraved on the test bar surface in a grid pattern before the deformation experiment (a) and the angle that is formed after the bars have been twisted is measured (b). Measurement is performed using a stereomicroscope (c).

Five different shear strain levels that correspond to different positions in the test bars were selected for the investigation (Table 2). The undeformed initial state of the material corresponds to zero shear strain. Five samples were taken from the test bars by precise cutting. The final bar radius tells that the compressive axial force decreases from $500 \mathrm{MPa}$ at the start of the test, down to $365 \mathrm{MPa}$ after 7 rotations. The hydrostatic pressure is naturally zero at the surface and low internally because of limited radial stress.

\begin{tabular}{|c|c|c|c|}
\hline Test bar & $\begin{array}{c}\text { Sample Position } r(\text { from } \\
\text { center line, in } \mathrm{mm})\end{array}$ & Final bar radius $R(\mathrm{~mm})$ & Shear strain $\gamma$ \\
\hline Undeformed & - & 5 & 0 \\
\hline 3 rotations & $3.28 \pm 0.3$ & 5.28 & $0.48 \pm 0.05$ \\
\hline 3 rotations & $4.53 \pm 0.3$ & 5.28 & $0.67 \pm 0.05$ \\
\hline 7 rotations & $3.85 \pm 0.3$ & 5.85 & $1.23 \pm 0.05$ \\
\hline 7 rotations & $5.1 \pm 0.3$ & 5.85 & $1.63 \pm 0.05$ \\
\hline
\end{tabular}

Table 2: Shear strain of the 5 test bar samples

\subsection{Microstructural characterization}

Standard grinding and electro-polishing (10\% perchloric acid in ethanol) procedures were used to prepare specimens for investigation in a JEOL 2000FX TEM. The ILS, and ferrite and cementite lamellar thickness were measured in TEM, taking care to ensure edge-on conditions to determine the thickness. The ILS value equals to the sum of ferrite lamellar thickness and cementite lamellar thickness. For each sample, data was collected from 30 measurements on randomly chosen areas, in order to get a macroscopic average value taking into account a microscopic local area for each measurement. Dislocation configurations and densities were examined using a JEOL 2000FX TEM at $200 \mathrm{kV}$. The dislocation density is determined by the line intersection method 


\section{ACCEPTED MANUSCRIPT}

applied to TEM micrographs. In addition, in order to reduce the TEM foil thickness effect on the dislocation configurations and densities, the measurements were performed at places with the thickness $>100 \mathrm{~nm}$.

\subsection{Micro-hardness measurements}

The Vickers microhardness measurements were conducted on a Struers DuraScan 70 hardness machine, using a $40 \mathrm{x}$ magnification lens and $500 \mathrm{~g}$ load (HV0.5). Circular samples were cut from the gauge section of the test bars. These were then sectioned as shown in figure 3 and indents were positioned in the cross section at different distances from the center line. Five indents per radial position from each side were measured. From these ten indents in total per position, an average hardness value was calculated.

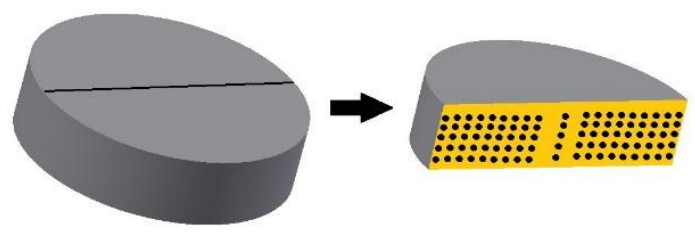

Figure 3: Samples taken from test bar gauge section and then cut along the black line. Hardness indents were positioned in the cross section as sketches.

\section{Results}

\subsection{Microstructure}

The initial pearlitic microstructure consists of alternating ferrite and cementite lamellae, as shown in Fig. 4. The initial ILS, thickness of ferrite and cementite lamellae are $233 \pm 39 \mathrm{~nm}, 193 \pm 30 \mathrm{~nm}$ and $40 \pm 6.5 \mathrm{~nm}$, respectively. These parameters decrease slowly as the shear strain increases up to around 1.2, after which the values decrease faster, as shown in Fig. 5 and Table 3. It is interesting that the cementite, which has brittle characteristics in the bulk form, shows a good deformability. The black arrows in the figure also show the dislocation structures in the ferrite lamellae, and the average size of a pearlite colony is around $8 \mu \mathrm{m}$. This initial structure relates to the rail rolling and subsequent cooling process used in the production of rails.

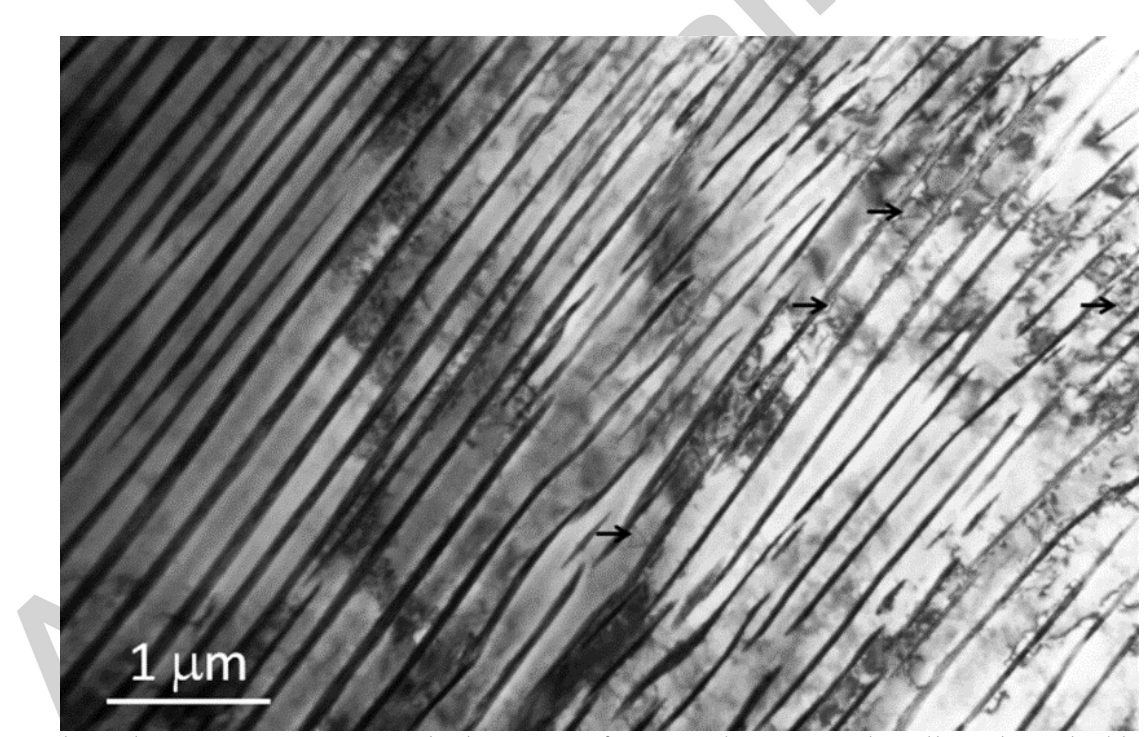

Figure 4: Initial pearlitic microstructure with alternating ferrite and cementite lamellae where the black arrows show examples of dislocations in the ferrite lamellae. 

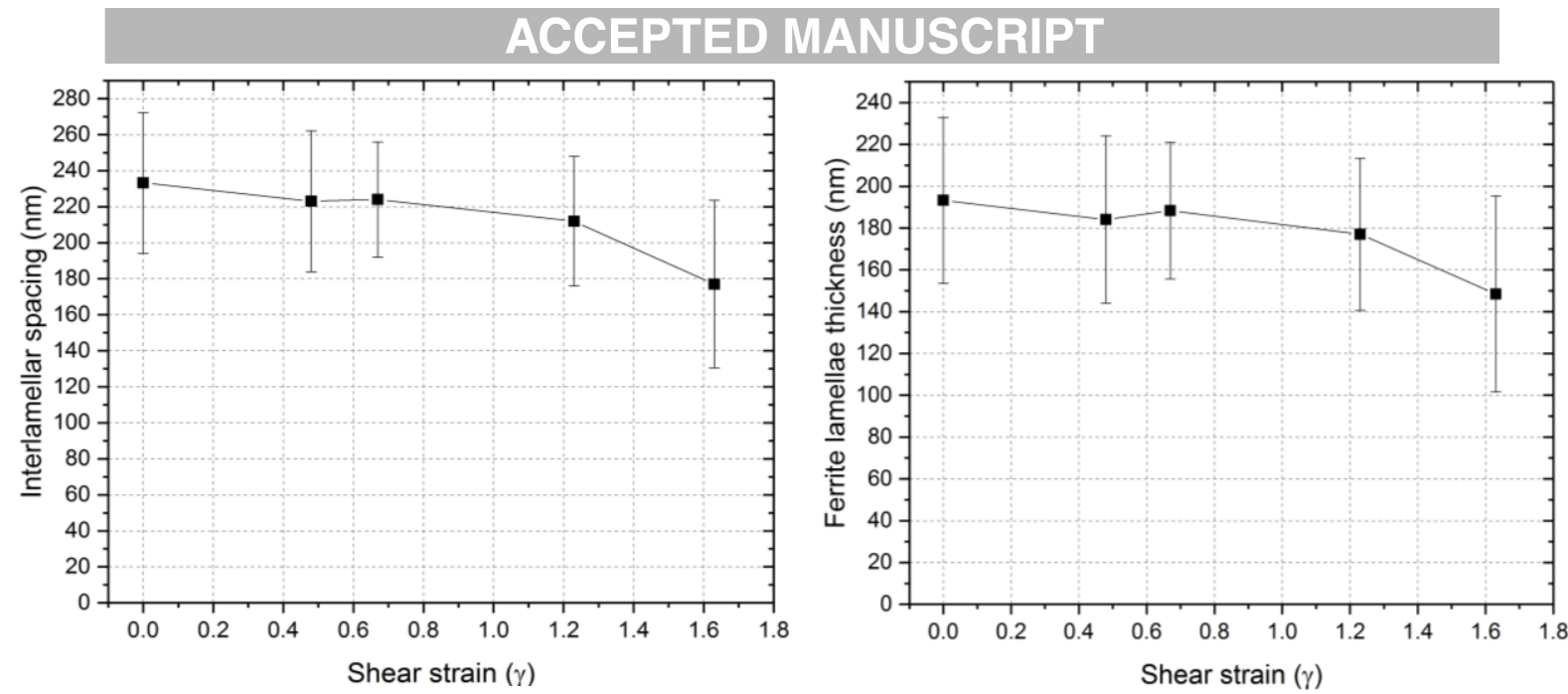

Figure 5: Interlamellar spacing and ferrite lamellae thickness versus the shear strain.

\begin{tabular}{|c|c|c|}
\hline Test bar & Shear strain & Thickness of cementite $(\mathrm{nm})$ \\
\hline Undeformed & 0 & $40 \pm 6.5$ \\
\hline 3 rotations & $0.48 \pm 0.05$ & $38.9 \pm 8$ \\
\hline 3 rotations & $0.67 \pm 0.05$ & $35.6 \pm 7.1$ \\
\hline 7 rotations & $1.23 \pm 0.05$ & $35 \pm 4.9$ \\
\hline 7 rotations & $1.63 \pm 0.05$ & $28.5 \pm 5.6$ \\
\hline
\end{tabular}

Table 3: Thickness of cementite lamellae versus the shear strain

\subsection{Dislocation configuration and density}

In the pearlitic steel with alternating ferrite and cementite lamellae, the deformation including yielding and plastic flow is largely controlled by processes occurring in ferrite, where the slip initially takes place in the ferrite lamellae, and then is transferred into the cementite lamellae [8]. Figure 6 shows the dislocations in the ferrite lamellae in the initial structure, with a dislocation density of $6 \times 10^{13} \mathrm{~m}^{-2}$. The random dislocation lines can be observed as a result of the phase transformation and many bulging-out dislocations can be observed from the ferrite/cementite interfaces which may have its cause in the elastic incompatibility stresses between the two phases [12, 13-15]. More dislocation loops with the two ends of the lines located at the ferrite/cementite interfaces have been observed at the shear strain of 0.48 , as shown in figure 7 . Calculation of the dislocation density gives a value of $2.4 \times 10^{14} \mathrm{~m}^{-2}$. Figure 8 shows the dislocation structure at a shear strain of 1.23 , and dislocation tangles are observed which indicates intense interactions between the dislocations. At this strain, the dislocation density is $5.3 \times 10^{14} \mathrm{~m}^{-2}$.

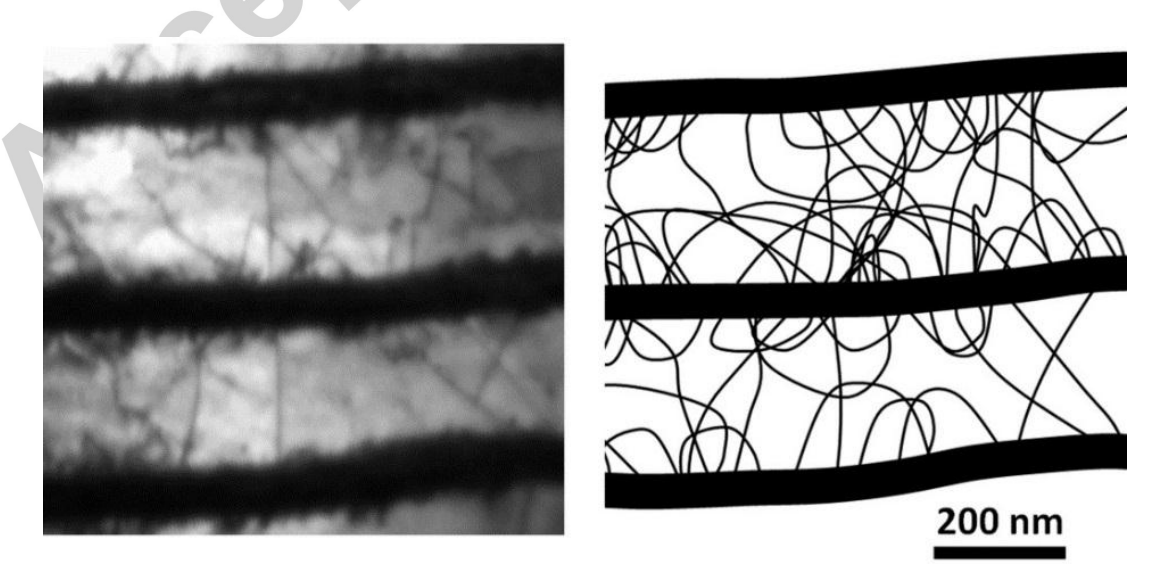

Figure 6: TEM micrograph of the initial sample and a sketch of the dislocation structure in the ferrite lamellae. The dislocation density is $6 \times 10^{13} \mathrm{~m}^{-2}$. 


\section{ACCEPTED MANUSCRIPT}
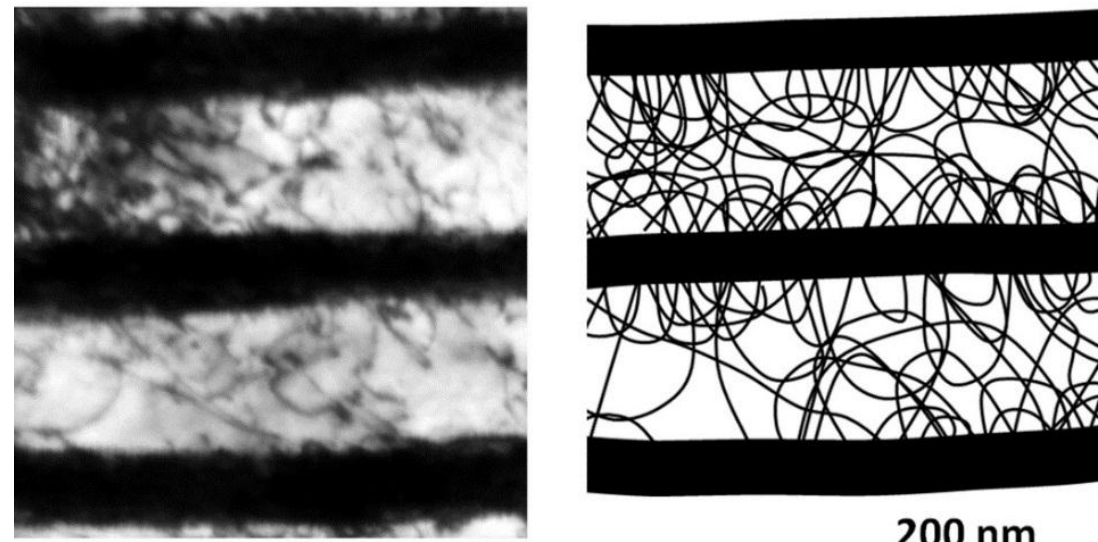

\section{$200 \mathrm{~nm}$}

Figure 7: TEM micrograph of the sample with a shear strain of 0.48 and a sketch of the dislocation structure in the ferrite lamellae. The dislocation density is $2.4 \times 10^{14} \mathrm{~m}^{-2}$.
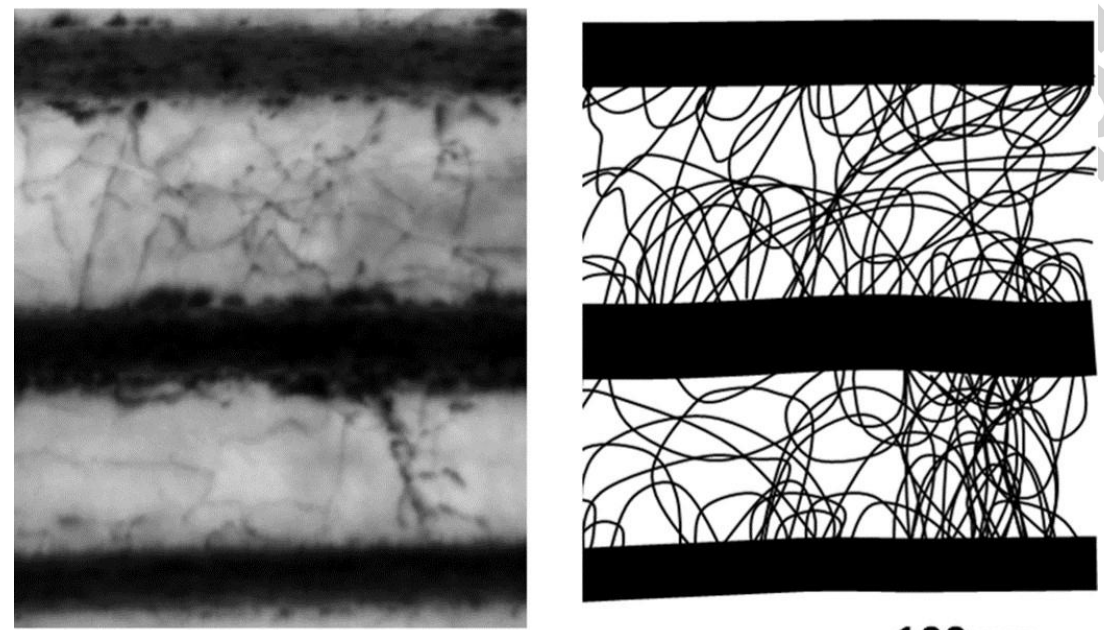

\section{$100 \mathrm{~nm}$}

Figure 8: TEM micrograph of the sample with a shear strain of 1.23 and a sketch of the dislocation structure in the ferrite lamellae. The dislocation density is $5.3 \times 10^{14} \mathrm{~m}^{-2}$.

The dislocation density is plotted in Fig. 9 versus the shear strain, showing an almost linear relationship.

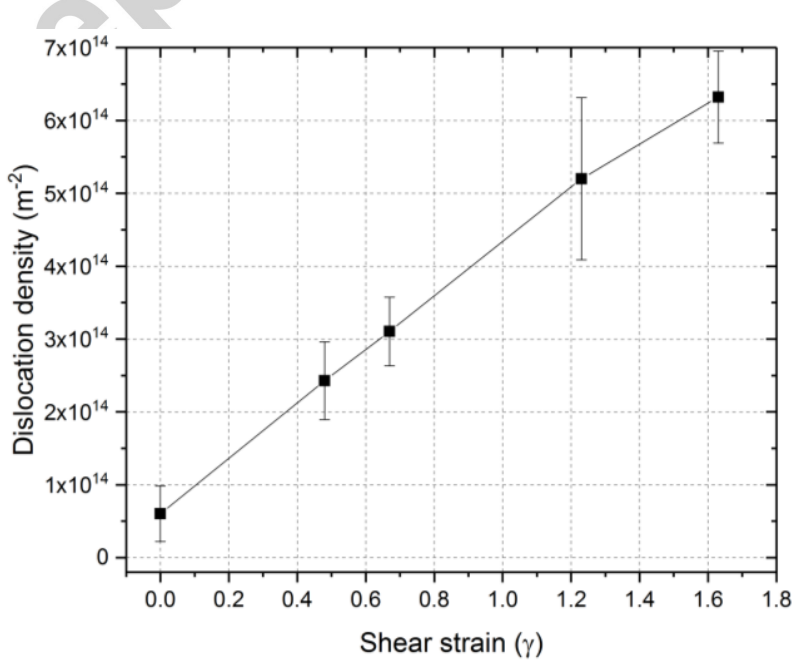

Figure 9: Dislocation density in the ferrite lamellae versus the shear strain. The error bar is determined using the values from around 30 areas. 


\subsection{Hardness measurements}

The hardness of the initial R260 material is $288 \mathrm{HV} 0.5$. The results of the hardness measurements of the pre-strained test bars are shown in figure 10. As expected, hardness increases with the shear strain.

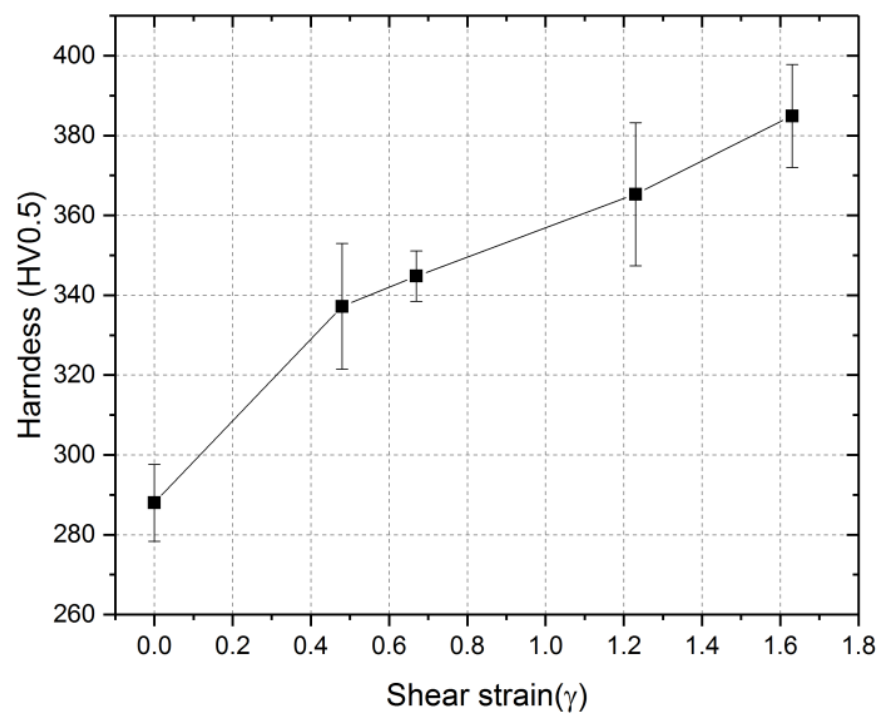

Figure 10: Hardness with increasing shear strain.

\subsection{Microstructure-strength relationships}

The microstructural characterization shows that below the shear strain 1.2, the change of ILS, thickness of ferrite and cementite is small, taking into account the standard deviation. The key factor contributing to the hardness increase with the shear strain is the increase of dislocation density in the ferrite lamellae. So, the following analysis focuses on the hardness/flow stress increase and the mechanisms behind.

The increases of flow stresses were determined experimentally from the nominal hardness measurements using the formula:

$$
\Delta H V_{0.5}=k \cdot \Delta \sigma_{\text {hardness }}
$$

with $k=3.3 \pm 0.4$, typical of deformed pearlitic steel in the range of low and medium strains [16]. This value is consistent with those in deformed metals where the lamellar dislocation structures form at medium and large strains [17-19], and hypoeutectoid steels having a wide range of compositions and a variety of microstructures [20]. Although the nano-sized lamellar structure has an anisotropic characteristic in one pearlite colony [21], this characteristic has been averaged and reduced by a much larger indent size, about 7 times the pearlite colony size in the present study.

The increase of flow stresses with the shear strain were also calculated from the deduction of the primary flow stress of initial material from the evaluated one from quantified microstructural parameters. From the microstructural observation, the flow stress of each shear strain was evaluated from two major microstructural parameters: the thickness of ferrite lamellae and dislocation density in the ferrite lamellae by considering two strengthening mechanisms: boundary strengthening related to the distance between the cementite lamellae, $\sigma(b)$ and dislocation strengthening related to the dislocation density in the ferrite lamellae, $\sigma(\rho)$. In the analysis of boundary strengthening, the contribution of cementite lamellae is estimated based on a Hall-Petch equation, where the barrier spacing is taken to be equal to the mean free path of dislocations, estimated to be twice the width of ferrite lamellae $[5,6,22,23]$. For the dislocation strengthening, the contribution of dislocation density to the strength is estimated as forest dislocation hardening proportional to the square root of the dislocation density. On the assumption that these strength contributions are linearly additive, thus the flow stress can be expressed as:

$$
\begin{gathered}
\sigma_{c a l}=\sigma_{0}+\sigma(b)+\sigma(\rho) \\
\sigma(b)=k \cdot(2 F)^{-0.5} \\
\sigma(\rho)=M \alpha G b \sqrt{\rho}
\end{gathered}
$$




\section{ACCEPTED MANUSCRIPT}

where $\sigma_{0}$ is the friction stress (60 MPa [5]), $k$ is the Hall-Petch constant for R260 pearlite steel, calculated using Eq. (3) and by inserting the flow stress $\left(\sigma_{0.2 \%}\right)$ of the initial material, $534.2 \mathrm{MPa}$ [24], $F$ is the thickness of ferrite lamellae, $M$ is the orientation factor, taken as $3, \alpha$ is a constant (0.24) [6], $G$ is the shear modulus of ferrite $(77.5 \mathrm{GPa})$ and $b$ is the Burgers vector $(0.248 \mathrm{~nm})$.

The flow stresses calculated based on the microstructural characterization and those measured by hardness are shown in Fig. 11. Taking into account the standard deviation from the microhardness measurements and the experimental uncertainties in the microstructural characterization behind the calculated flow stress increase, the agreement is good.

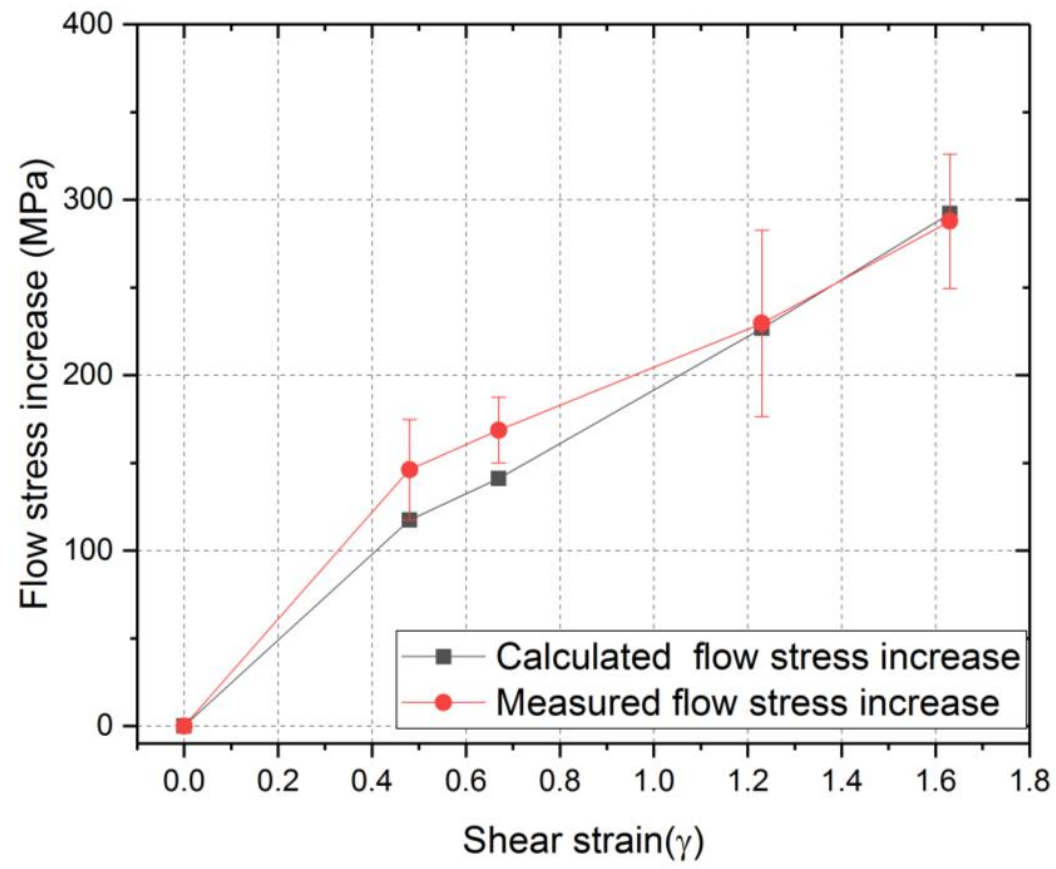

Figure 11: The calculated and measured flow stress increases from microstructural parameters and microhardness versus the shear strain, respectively.

\section{Discussion}

In the present study, the endeavor has been to develop the relationships among shear strain, microstructure and flow stress in pre-deformed test bars, to understand the materials behavior in the gradient deformed rail surface. The following discussion will focus on the microstructural evolution with the shear strain and strengthening mechanisms.

\subsection{Microstructural evolution with the shear strain}

At the present small and medium strains, the lamellar structure reorients; for example, the lamellae are almost vertical to the bar axis at the (sub)surface of the sample with the highest shear strain (7-turns, 1.63). This reorientation process also involves shear banding and pearlite colony curling, as in pearlitic steel wire drawing $[13,23,25]$. Such heterogeneities change the microstructural morphology and may also affect the arrangement and density of dislocations in the ferrite lamellae. However, the characterization of such heterogeneities is for further research, as it will require a fairly detailed characterization at different positions in the bars. The following analysis will therefore be based on the assumption of a lamellar structure and values for the interlamellar spacing and the dislocation density will be the average values for all structures. This averaging also allows the present data to be compared with data reported in the literature of deformed pearlite.

\subsection{Strengthening mechanisms}

A key finding is the evolution of the dislocation structure and dislocation density with the shear strain. The dislocations are organized from a two-dimensional bulging-out state to a three-dimensional tangle state. This observation of dislocation storage in the ferrite lamellae has led to the application of the Hall-Petch relationship for the local flow stress, where boundary strengthening and dislocation strengthening are taken into account. Good agreement between the calculated and the evaluated flow stress increase has been found. 


\section{ACCEPTED MANUSCRIPT}

The boundary strengthening due to the cementite lamellae based on the Hall-Petch relationship have been analyzed in earlier studies $[6,26]$. This application is based on the assumption that yield takes place as the stress in front of a dislocation pile-up reaches the barrier strength or operates a dislocation source in the neighboring grains [27]. The extrapolation of grain boundaries to cementite lamellae will then focus on the dislocation pile-ups in the ferrite lamellae. For a pile-up of screw or edge dislocations, the number of dislocations in a pile-up has been related to the applied stress $(\sigma)$ and the pile-up length $\left(L_{p}\right)$ :

$$
n=(c \pi / G b) \cdot(\sigma / M) \cdot L_{p}
$$

where $c=0.835$ as an average between edge and screw dislocations. For $L_{p}=2 F$ and by inserting the numbers in Section 3, it is suggested that a pile-up of about 16 dislocations in the ferrite lamellae with a thickness around $180 \mathrm{~nm}$ is possible.

The contribution of dislocation strengthening to the local flow stress has been calculated as forest hardening proportional to the square root of dislocation density. An alternative to this mechanism is the Orowan stress to propagate single dislocation loops through the interface and the dislocations are deposited at or near the interfaces [28]. This is not the case in the present observation as the dislocation density is high both in the ferrite lamellae and at the ferrite-cementite interfaces and the 3-D dislocation tangles has formed at the medium shear strains.

However, for larger strains typically found even closer to the rail surfaces, the full understanding and integration of the strengthening mechanisms in the present structure-strength model may encompass atomistic characterization such as 3D atom probe tomography [29].

\section{Conclusions}

To investigate the mechanical response of the surface layer of railway rails after run-in, test bars of the pearlitic rail steel grade R260 were deformed using a bi-axial torsion-compression machine up to a medium shear strain of 1.63. A thorough investigation of how the microstructure evolves with shear strains and of how the microstructure affects the mechanical properties has been performed via detailed microstructural and mechanical characterization and quantification by TEM and microhardness. The conclusions are the following.

1. The interlamellar spacing, and the thickness of ferrite and cementite lamellae decrease very slowly with the increase of shear strain up to around 1.2, after which a faster decrease has been observed.

2. The dislocation structure changes from single, straight dislocation lines and dislocation loops from ferrite/cementite interfaces in the initial state to dislocation tangles at the highest shear strains, and the dislocation density increases from $6.0 \times 10^{13} \mathrm{~m}^{-2}$ in the initial state to $5.3 \times 10^{14} \mathrm{~m}^{-2}$ at a shear strain of 1.63. This increase of dislocation density is the key factor behind the increase of local hardness/flow stress with shear strain.

3. The microstructural characterization suggests two major strengthening mechanisms: boundary strengthening and dislocation strengthening. The individual contributions from the two strengthening mechanisms to the local strength have been estimated based on the experimentally quantified structural parameters, and on the assumption that these contributions are linearly additive. Good agreement has been found between the calculated flow stresses increase from quantified structural parameters and the measured flow stresses increase from microhardness, which is underpinned by a discussion of the two strengthening mechanisms in $\sim 200 \mathrm{~nm}$ lamellar structures.

\section{Acknowledgements}

D.N. and J.A. gratefully thank the National Centre of Excellence CHARMEC (Chalmers Railway Mechanics, www.charmec.chalmers.se). Their work is partly financed within the European Horizon 2020 Joint Technology Initiative Shift2Rail through contract No. 730841. X.Z. gratefully acknowledge the support from the Innovation Fund Denmark to the Intelligent Quality Assessment of Railway Switches and Crossings (INTELLISWITCH) project. The authors also thank Prof. N. Hansen and Prof. D. Juul Jensen for their valuable comments during preparation of the manuscript.

\section{References}

[1] F. A. M. Alwahdi, A. Kapoor, F. J. Franklin, Subsurface microstructural analysis and mechanical properties of pearlitic rail steels in service, Wear 302 (1-2) (2013) 1453-1460. doi:10.1016/j.wear.2012.12.058.

[2] K. Cvetkovski, J. Ahlström, Characterisation of plastic deformation and thermal softening of the surface layer of railway passenger wheel treads, Wear 300 (1-2) (2013) 200-204. doi:10.1016/j.wear.2013.01.094.

[3] K. A. Meyer, D. Nikas, J. Ahlström, Microstructure and mechanical properties of the running band in a 


\section{ACCEPTED MANUSCRIPT}

pearlitic rail steel: Comparison between biaxially deformed steel and field samples, Wear 396-397 (August 2017) (2018) 12-21. doi:10.1016/j.wear.2017.11.003.

[4] X. Zhang, A. Godfrey, N. Hansen, X. Huang, W. Liu, Q. Liu, Evolution of cementite morphology in pearlitic steel wire during wet wire drawing, Materials Characterization 61 (1) (2010) 65-72. doi:10.1016/j.matchar.2009.10.007.

[5] X. Zhang, N. Hansen, A. Godfrey, X. Huang, Dislocation-based plasticity and strengthening mechanisms in sub$20 \mathrm{~nm}$ lamellar structures in pearlitic steel wire, Acta Materialia 114 (2016) 176-183. doi:10.1016/j.actamat.2016.04.040.

[6] X. Zhang, A. Godfrey, X. Huang, N. Hansen, Q. Liu, Microstructure and strengthening mechanisms in cold-drawn pearlitic steel wire, Acta Materialia 59 (9) (2011) 3422-3430. doi:10.1016/j.actamat.2011.02.017.

[7] K.N. Zhu, A. Godfrey, N.Hansen, X. Zhang, Microstructure and mechanical strength of near- and sub-micrometre grain size copper prepared by spark plasma sintering, Materials \& Design 117 (2017) 95-103.

[8] S. Chen, J. Hu, X. Zhang, H. Dong, W. Cao, High Ductility and Toughness of a Micro-duplex Medium-Mn Steel in a Large Temperature Range from $-196^{\circ} \mathrm{C}$ to $200^{\circ} \mathrm{C}$, Journal of Iron and Steel Research, International 22 (2015) 1126-1130.

[9] G.H. Fan, Q.W. Wang, Y. Du, L. Geng, W. Hu, X. Zhang, Y.D. Huang, Producing laminated NiAl with bimodal distribution of grain size by solid-liquid reaction treatment, Materials Science and Engineering A 590 (2014) 318-322.

[10] H. P. Stüwe, Equivalent strains in severe plastic deformation, Advanced Engineering Materials 5 (5) (2003) 291 295. doi:10.1002/adem.200310085.

[11] R. Pippan, F. Wetscher, M. Hafok, A. Vorhauer, I. Sabirov, The limits of refinement by severe plastic deformation, Advanced Engineering Materials 8 (11) (2006) 1046-1056. doi:10.1002/adem.200600133.

[12] X. Zhang, A. Godfrey, N. Hansen, X. Huang, Hierarchical structures in cold-drawn pearlitic steel wire, Acta Materialia 61 (13) (2013) 4898-4909. doi:10.1016/j.actamat.2013.04.057.

[13] X. Zhang, A. Godfrey, W. Liu, Q. Liu, Study on dislocation slips in ferrite and deformation of cementite in cold drawn pearlitic steel wires from medium to high strain, Materials Science and Technology 27 (2) (2011) 562-567. doi:10.1179/026708309X12512744154405.

[14] M. Dollar, I. M. Bernstein, A. W. Thompson, Influence of deformation substructure on flow and fracture of fully pearlitic steel, Acta Metallurgica 36 (2) (1988) 311-320. doi:10.1016/0001-6160(88)90008-9.

[15] M. Guziewski, S. P. Coleman, C. R. Weinberger, Atomistic investigation into the mechanical properties of the ferrite- cementite interface: The Bagaryatskii orientation, Acta Materialia 144 (2018) 656-665. doi:10.1016/j.actamat.2017.10.070.

[16] X. Zhang, Quantitative investigation of microstructural evolution during the cold wire-drawing of a pearlitic steel wire and its relationship with mechanical properties, Ph.D. thesis, Tsinghua University, Beijing (2009).

[17] M.A. Meyers, K.K. Chawla, Mechanical behavior of materials, Cambridge University Press, Cambridge, UK (2009), p. 223-224.

[18] X. Zhang, N. Hansen, Y. Gao, X. Huang, Hall-Petch and dislocation strengthening in graded nanostructured steel, Acta Materialia 60 (2012) 5933-5943.

[19] X. Zhang, N. Hansen, C. V. Nielsen, Local microstructure and flow stress in deformed metals, 2017 IOP Conf. Ser.: Mater. Sci. Eng. 219012053.

[20] E. J. Pavlina, C. J. Van Tyne, Correlation of Yield Strength and Tensile Strength with Hardness for Steels, Journal of Materials Engineering and Performance 17 (2008) 888-893.

[21] M. Kapp, A. Hohenwarter, S. Wurster,B. Wang, R. Pippan, Anisotropic deformation characteristics of an ultrafineand nanolamellar pearlitic steel, Acta Mater. 106 (2016) 239-248.

[22] M. Gensamer, E. B. Pearsall, W. S. Pellini, J. R. Low, The tensile properties of steel, Transactions of American Society for Metals 30 (1942) 983-1019.

[23] X. Zhang, N. Hansen, A. Godfrey, X. Huang, Structure and strength of sub-100nm lamellar structures in colddrawn pearlitic steel wire, Materials Science and Technology (2018). doi:10.1080/02670836.2018.1440155.

[24] K.A. Meyer, M. Ekh, J. Ahlström, Modeling of kinematic hardening at large biaxial deformations in pearlitic rail steel. International Journal of Solids and Structures, 130-131 (2018), p.122-132.

[25] G. Langford, Deformation of pearlite, Metallurgical Transactions A 8 (6) (1977) 861-875. doi:10.1007/BF02661567.

[26] J.D. Embury, R.M. Fisher, The structure and properties of drawn pearlite, Acta Metall. 14 (1966), p.147-159.

[27] J. D. Eshelby, F. C. Frank, F. R. N. Nabarro, The equilibtium of linear arrays of dislocations, Phil. Mag. 42 (1951) 351-364.

[28] J.D. Embury, J.P. Hirth, On dislocation storage and the mechanical response of fine scale microstructures, Acta Metall. Mater., 42 (1994), p. 2051-2056.

[29] Y.J. Li, P. Choi, C. Borchers, Y.Z. Chen, S. Goto, D. Raabe, R. Kirchheim, Atom probe tomography characterization of heavily cold drawn pearlitic steel wire, Ultramicroscopy, 111(2011), P.628-632. 